\title{
УДК 553.1
}

\section{С. П. Бардаханов, Д. Ю. Труфанов, А. П. Завьялов}

\author{
Институт теоретической и прикладной механики \\ им. С. А. Христиановича СО РАН \\ ул. Институтская, 4/1, Новосибирск, 630090, Россия \\ trufanov@itam.nsc.ru
}

\section{НОВЫЕ СВОЙСТВА СПЛОШНОЙ СРЕДЫ - ГАЗЫ С ВЫСОКОЙ КОНЦЕНТРАЦИЕЙ НАНОЧАСТИЦ *}

\begin{abstract}
Представлен обзор работ, результаты которых свидетельствуют о необходимости постановки проблемы описания новых типов сплошных сред, содержащих большие концентрации наноразмерных частиц. Подчеркивается важность этой проблемы для понимания современных технологических процессов: образование нанопорошков различной природы и использование компонентов в виде наночастиц, концентрация которых достигает существенных значений. Рассмотрены примеры практически важных течений порошковых материалов. Предложено понятие новой сплошной среды при высокой концентрации наночастиц, и применен термоанемометрический метод.
\end{abstract}

Ключевые слова: нанопорошок, течение газов, течение сплошной среды, гранулированная среда.

\section{Новый тип сплошной среды}

Основными методами получения наноразмерных порошков являются газофазные методы, в общем случае этот процесс заключается в охлаждении паров в газовом потоке, достаточно быстром, чтобы предотвратить рост частиц. Например, один из основных методов получения нанопорошков диоксида кремния заключается в сжигании тетрахлорида кремния в кислородно-водородной горелке [1]. В результате этой реакции в поток попадают пары диоксида кремния, которые, быстро охлаждаясь, образуют частицы наноразмерного масштаба. В каче- стве альтернативы такому методу можно использовать прямое испарение диоксида кремния. В описываемых экспериментальных исследованиях дополнительно использовались нанопорошки, для получения которых в качестве источника нагрева использовался ускоритель электронов [2]. В этих методах частицы улавливаются похожим образом: после реактора, частицы пролетают достаточно продолжительное время, чтобы они успели в результате соударений друг с другом образовать агломераты. Обладающие необходимой массой конгломераты из частиц улавливаются циклоном из потока. В установке, испаряющей сырье электрон-

\footnotetext{
* Авторы выражают благодарность за всестороннюю поддержку, помощь в проведении экспериментов Y. U. Han, D. Y. Lee, S. W. Joo, M. E. Djuraeva, E. Ivanov, O. Y. Korkina, Y. H. Lim, C. А. Козлову, В. В. Ларичкину, А. П. Фатьянову, А. Ковелаеву, В. В. Обанину, В. В. Козлову, В. Я. Рудяку, А. Б. Семенову, а также за плодотворное обсуждение направлений и результатов исследований.

Д. Ю. Труфанов выражает благодарность Российскому фонду фундаментальных исследований за поддержку в рамках гранта № 16-31-60076.

С. П. Бардаханов благодарит Минобрнауки РФ за поддержку в рамках проектной части Государственного задания в сфере научной деятельности по заданию No. 16.1930.2014/K.
}

Бардаханов С. П., Труфанов Д. Ю., Завьялов А. П. Новые свойства сплошной среды - газы с высокой концентрацией наночастиц // Сибирский физический журнал. 2017. Т. 12, № 2. С. 100-110. 
ным пучком, при производительности 7 кг/ч расходуется около $700 \mathrm{~m}^{3}$ воздуха. Это соответствует потоку, в котором концентрация наночастиц составляет примерно $1 \%$ по массе. В промышленном производстве фирмы «Evonik» эта концентрация может достигать $6 \%$. Это достаточно насыщенные наночастицами потоки газа.

После производства порошков возникает необходимость их доставки до места непосредственного использования. При решении таких задач, как движение по трубам, расфасовка и дозирование порошка при использовании его в качестве материала на производстве, мы сталкиваемся с проблемой описания течения гранулированной среды.

Модель сплошной среды применима тогда, когда для решения той или иной задачи мы можем пренебречь внутренним устройством среды и рассматривать ее как некое поле плотностей с соответствующим векторным полем скоростей. Характерными примерами сплошных сред являются газ, жидкость, плазма и твердое тело. Во всех случаях применения модели сплошной среды масштаб, характеризующий ее внутреннюю структуру (длина свободного пробега, в газе или жидкости длина связей в кристаллической решетке твердого тела), существенно меньше масштабов рассматриваемой задачи. Особенностью наноразмерного порошка является то, что его характерный масштаб также мал.

С одной стороны, нанопорошок является сыпучим твердым телом, таким же как, например, строительный песок. Но нанопорошок имеет принципиально другую структуру по сравнению с сыпучими телами, состоящими из крупных частиц. В отличие от них в нанопорошке основной составляющей по объему является воздух, в некоторых порошках воздух может составлять более $98 \%$ объема, оставляя на твердую фазу менее $2 \%$. Чтобы занимать такой большой объем при малом количестве твердой фазы, частицы должны выстраивать агломераты, которые были бы достаточно прочные чтобы держать вес агломератов, находящихся над ними. Объем заполненный такими агломератами должен представлять собой высокопористую сетку из наночастиц, пустоты которой заполнены воздухом, причем воздух в таких порах не будет сплошной средой. Число Кнудсена для течения воздуха сквозь поры в нанопорошке будет составлять 2-9, что делает почти невозможным такое течение из-за огромного сопротивления порошка, которое больше, чем силы, необходимые для передвижения пористой среды. Характерный масштаб, при котором можно подробно рассмотреть структуру нанопорошка, сопоставим с длиной свободного пробега молекул газа. Надо принять во внимание и количество частиц порошка в сравнении с количеством молекул газа. Например, для порошка диоксида кремния Т-20 при насыпной плотности 50 г/л он будет содержать $1,2 \cdot 10^{17}$ первичных частиц порошка и $2,4 \cdot 10^{19}$ молекул воздуха. С другой стороны, про такой порошок нельзя сказать, что он полностью подобен аэрозолю, так как в состоянии покоя все частицы прочно связаны друг с другом и не участвуют в броуновском движении.

Учитывая это, мы можем предположить, что моделирование движения нанопорошков аналогично движению других сыпучих тел может привести к неправильному результату или неверному пониманию процесса. Из этих соображений можно сделать вывод, что нанопорошок, воздействуя с макроскопическими объектами при своем течении, должен обладать в том числе и свойствами, которыми обладает неразрывно связанный с ним воздух.

Теперь вернемся к масштабу рассматриваемых задач. Например, не сложно понять, что нить датчика термоанемометра, диаметр которого составляет несколько микрон, структура, средний масштаб которой сопоставим с длиной свободного пробега, - макроскопический объект, который будет взаимодействовать одновременно с тысячами частиц порошка. Эти соображения позволяют нам предположить, что датчик будет распознавать порошок как сплошную среду.

\section{Методы исследований течений нанопорошков}

Общей проблемой для изучения движения всех сыпучих тел является недостаток средств получения данных о течении. Так, например, PIV не в состоянии отслеживать такое множество частиц. Кроме того, лазеру необходимо просвечивать достаточно большую толщину потока, соответственно, помимо геометрических ограничений на поток, это существенно усложняет расчет скоростей из-за многократных рассеяний. Извест- 
ны также работы, в которых использовалась электродинамическая томография [3] для осевых скоростных измерений вблизи стены конвейера с шариками, движущимися под действием силы тяжести. Более новые исследования порошковых потоков проведены с использованием рентгеновских лучей [4], допплеровской лазерной анемометрии [5] и магнитного резонанса [6], но каждый из этих методов имеет ограничения в применении. Существенного прогресса в понимании процессов, проходящих в сыпучей среде, они не добились. Ниже приведены оценочные эксперименты, показывающие сходство течения нанопорошков с течениями классических сплошных сред.

\section{«Сообщุаюшиеся сосудыл»}

Наблюдения за течениями в трубах малого диаметра, заполненных нанопорошком, в том числе U-образных, под действием давления, создаваемого либо воздухом, либо порошком, показали, что имеется по крайней мере внешнее подобие такого течения и течения жидкости в трубках. Мог реализовываться режим как «снарядного» течения, так и сплошного движения. Справедливости ради нужно сказать, что полностью одинаковый уровень положения «менисков» не достигался. Как уже указывалось в начале настоящего обзора, плотность нанопорошков значительно ниже плотности, например, воды, поэтому, возможно, малый уровень «давления», создаваемого нанопорошком, и сильное поверхностное взаимодействие порошков со стенками трубок не позволяли достичь полноценного состояния «сообщающихся сосудов». Однако некоторые данные указывают, что выбор соответствующего нанопорошка, диаметра и радиуса кривизны трубок и других параметров может позволить достигнуть полной аналогии.

\section{Течение под действием вентилятора}

В промышленности широко используются шнековые устройства для перемещения тяжелых гранулированных сред, вентиляторные же устройства ввиду больших энергозатрат практически не применяются. В то же время пневмотранспорт является распространенным средством в производствах ультрадисперсных и нанопорошков.
Для выяснения возможности перемещения порошков при помощи вентилятора внутрь стеклянной трубы устанавливался электродвигатель с вентилятором. Было получено два режима течения: режим транспортировки порошка как сплошной среды (как в шнековых устройствах для тяжелых сыпучих сред) при относительно малом энергоподводе к вентилятору и как запыленного воздуха с образованием «дюн», когда устанавливалась относительно высокая скорость вращения вентилятора. В обоих случаях реализовывалась винтовая картина течения. Авторы усматривают в этом случае аналогию, например, с экспериментами K. Takahashi [7], который провел разделение режимов течения в наклонных лотках по скорости движения слоя засыпки.

\section{Движение мара в нанопорошке}

под действием силь тяжести

Одним из методов, позволяющих оценить свойства нанопорошка, подобные свойствам жидкостей и газов, является измерение скорости, с которой падает шарик сквозь среду. Нанопорошок ввиду своей структуры существенно отличается от макроскопических сыпучих веществ. Так, например, стальной шарик, брошенный в песок или другую плотную гранулированную среду, останется у ее поверхности, нанопорошок же позволит шарику упасть на самое дно емкости. В этом эксперименте у нас есть длина пройденного пути и временной промежуток от начала падения до соприкосновения шарика со дном. По этим данным по закону Стокса можно оценить вязкость порошка. Из полученных таким образом оценок можно сделать вывод, что нанопорошок примерно на порядок более вязкий, чем вода, в то же время на порядок менее вязкий, чем распространенные в промышленности жидкие масла. Кроме того, эта установка позволяет получать сравнительные вязкости порошков различных веществ. Так, например, было показано, что вязкость нанопорошков диоксида кремния выше, чем вязкость порошка оксида алюминия.

\section{Течение Куэтта}

Следующим шагом в моделировании течений нанопорошков была реализация тече- 
ния, похожего на течение Куэтта. Для этого использовались две пластины площадью 0,6 м², расположенные параллельно друг другу на расстоянии 10 мм. Верхняя пластина могла свободно двигаться по направляющим. Было замечено, что при движении одной пластины относительно другой слой порошка увлекался за движущейся пластиной, но провести точные измерения не удалось из-за непродолжительного времени эксперимента. Это было связано с двумя особенностями. Во-первых, пластины двигались относительно друг друга достаточно непродолжительное время, и не было уверенности, что поток успевает установиться. Во-вторых, из-за непараллельности пластин и пластичности порошка со временем между верхней пластиной и порошком образовывались воздушные прослойки, и контакт с пластиной терялся

\section{Термоанемометрические измерения в порошковых средах}

Предыдущие эксперименты могли дать только качественное сравнение картин течений. Для получения более точных данных о движении и векторном поле скоростей порошковой среды был применен датчик термоанемометра [8].

\section{Течение в воронке \\ под действием колебаний расхода}

Скорость истечения гранулированных материалов из воронкообразных бункеров, внутри них, а также характер движения в лотках являлись предметом ряда экспериментальных исследований и нескольких теоретических работ, например, K. Ridgway [9], C. Campbell [10]. Снова заметим, что абсолютное большинство прежних исследований касалось задач медленного оседания или течения гранулированных материалов с крупными частицами, а в реальности минимальные их размеры соответствовали нескольким сотням микрон. Кинематика поля скоростей была связана с каким-либо первоначально определенным полем напряжений. Предполагалось, что они не зависят от скоростей деформации. Хотя данное предположение может соответствовать некоторым очень медленным гранулированным течениям (например, в верхней части бункеров и воронок), инерционные эффекты, а также влияние скоростей деформаций существенны для течений с крупными частицами.

A. Suzuki, H. Takahashi, T. Tanaka [11] исследовали влияние вибрационного воздействия на течения крупных сыпучих материалов в воронках. Заметим, в дополнение к параграфу о движении шаров в нанопорошке, что одновременно они же попытались использовать наклонный лоток в качестве вискозиметра для гранулированных материалов. В таких быстрых сдвиговых течениях, как и в медленных «квазистатических», одной из центральных проблем теоретического характера является разработка определяющих уравнений, которые могли бы использоваться для описания течений в различных практических установках. Для течений же нанопорошков как раз характерны относительно малые уровни инерционных сил. Причем в отличие от течений «грубодисперсных» сыпучих сред, для которых отсутствуют методы количественного измерений структуры колебаний внутри течения, применение термоанемометрического метода позволяет получить данные как о средних, так и о флуктуационных параметрах движений именно внутри гранулированной среды. Поэтому была создана установка для соответствующих исследований, схема которой приведена на рис. 1.

В воронку насыпался нанопорошок, в нижнюю часть воронки насосом подавался пульсирующий воздух с определенным расходом. В результате в среде нанопорошка наблюдалась пульсирующая струя, в которой можно было регистрировать путем перемещения координатника средние скорости и флуктуации скорости в любой области объема нанопорошка (рис. 2). Исследования

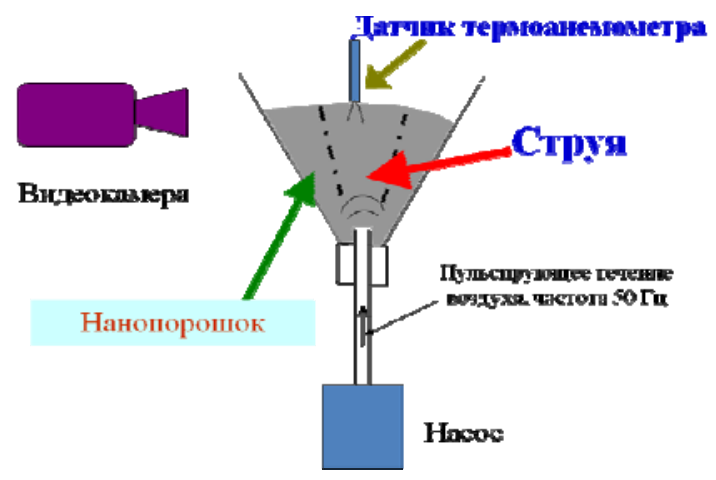

Puc. 1. Схема установки 


\section{Hot-wire signal 1}

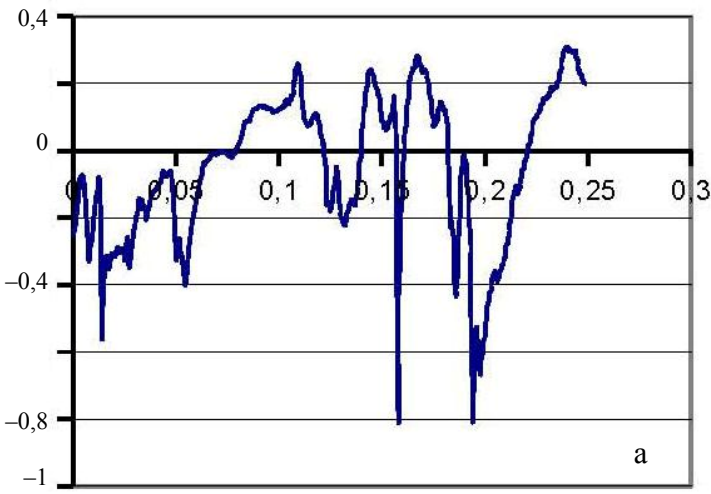

Hot-wire signal 2

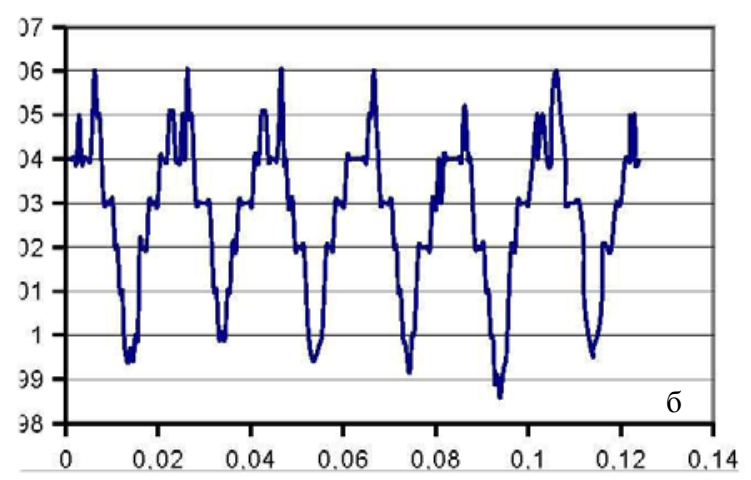

Hot-wire signal 3

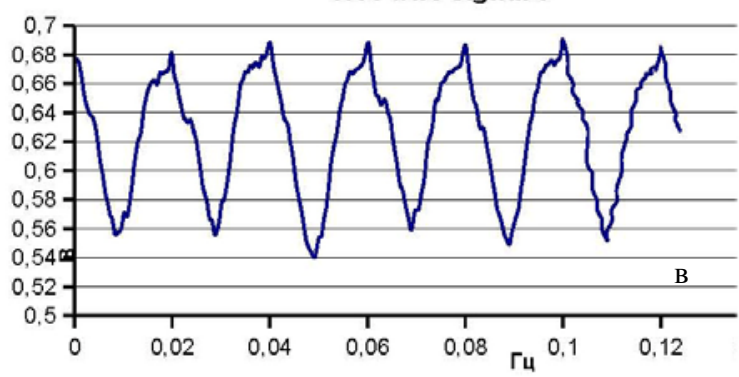

Puc. 2. Реализации сигнала пульсаций скорости в различных областях пульсирующей струи в нанопорошке

в данной постановке могут иметь большое значение для проверки различных теоретических подходов в механике гранулированных сред.

\section{Течение по наклонной плоскости} под действием акустического поля

K. Takahashi [12] еще в 1930-е гг. изучал гравитационное течение различных сухих песков в прямом лотке прямоугольного поперечного сечения при разных углах его на- клона. Он отметил два режима течения: один состоит в том, что верхний тонкий слой частиц течет над их стационарным слоем, покрывающим дно лотка, и второй в том, что все частицы движутся, причем каждая из них хаотически флуктуирует и существенно взаимодействует с соседними. Скорость частиц (оцененная по их траектории), покидающих выходное сечение канала, быстро возрастала с увеличением угла наклона при первом режиме течения и гораздо более медленно при втором.

K. Ridgway, R. Rupp [9] исследовали течение различных песков вниз по латунному лотку прямоугольного поперечного сечения. Частицы трех различных размеров в диапазонах 250-355, 420-500, 758-850 мкм были получены путем просеивания. Скорость движения частиц определялась с помощью высокоскоростной кинокамеры путем их съемки сверху на свободной поверхности и снизу через стеклянное окно в дне лотка. По его глубине не было обнаружено градиента скорости.

Нужно заметить, что реологические особенности нанопорошков таковы, что для возникновения течения нужны значительно бо́льшие углы наклона лотков по сравнению с крупнодисперсными порошками. Причиной этого являются отмеченные выше очень малые насыпные плотности нанопорошков и определяющее значение поверхностных сил на частицах нанопорошков, например, сильная агломерация наночастиц и другие характерные реологические особенности, обусловленные физико-химическими свойствами наночастиц.

Для исследования влияния возмущений на возникновение движения нанопорошков в наклонных лотках была создана установка, схема которой показана на рис. 3 .

Нанопорошок насыпался в лоток, причем углы наклона выбирались значительно меньшими, чем те, при которых возникло бы «самопроизвольное» течение. Затем включался громкоговоритель на определенных частотах. Звук и частоты контролировались микрофоном. В слой нанопорошка помещался датчик термоанемометра, который регистрировал флуктуации скорости в нанопорошке, двигавшемся в направлении, указанном стрелкой. Монохроматичность акустического возбуждения и его уровень контролировались на анализаторе сигналов, в то же время сигнал с термоанемометриче- 
Громкоговоритель

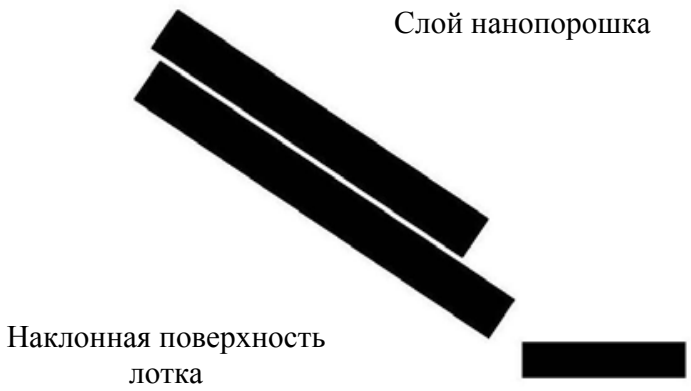

Puc. 3. Схема установки для исследования течений нанопорошков в наклонных лотках под действием акустического поля

ской аппаратуры показал, что в течении могли возникать дополнительные частоты, обусловленные нелинейными процессами в движущемся нанопорошке.

При одинаковом уровне акустического воздействия интенсивность движения в слое могла существенно отличаться, причем значительно. Это позволяет предположить, что для данного типа течения может существовать диапазон частот, в котором отклик потока максимальный. В целом интегральный уровень звука лежал в диапазоне 80-90 дБ. При превышении этого уровня движение нанопорошка становилось особенно интенсивным, с возникновением аэрозольного тумана в области движения.

\section{Сопоставление с течением газов}

\section{Течение нанопорошка}

под действием силь тяжести

в вертикальном канале

Первые достаточно точные результаты термоанемометрии в течении наноразмерного порошка были получены в вертикальном канале [13]. Установка представляла собой вертикальную трубу с возможностью следить за уровнем порошка в ней, в нижней части трубы устанавливался датчик термоанемометра. Для тарировки датчика использовалась видеокамера, с помощью которой определялась скорость верхней границы на- нопорошка. На рис. 4 представлено сопоставление скорости, измеренной при помощи видеокамеры, и сигнала с датчика термоанемометра, видно что зависимости подобны.

Далее датчик перемещался вдоль радиальной составляющей канала, и определялась скорость. Выяснилось, что достаточно удаленное от стенок трубы «ядро» течения имеет одинаковую скорость и фактически движется как одно целое, но с приближением к стенкам трубы скорость падала, а флуктуации росли. Такой характер течения может быть следствием того, что эксперимент проходит слишком быстро, время пуска не больше 2,5 c, в результате чего течение не успевает устанавливаться. Кроме того, способ тарировки и наличие существенных погрешностей не позволял сравнить данные для одного датчика с тарировкой в воздухе.

Чтобы сравнить взаимодействие нанопорошка и воздуха, на датчик термоанемометра была проведена тарировка измерительного прибора в двух средах [14]. В экспериментах использовался термоанемометр фирмы DISA55B01, нить датчика термоанемометра имела длину 1,5 мм и диаметр 6 мкм, на мосте устанавливался перегрев 0,8. В воздухе тарировка проходила в ламинарной дозвуковой трубе МТ-324 по стандартной методике. Для тарировки же датчика в нанопорошке была создана специальная уста- 

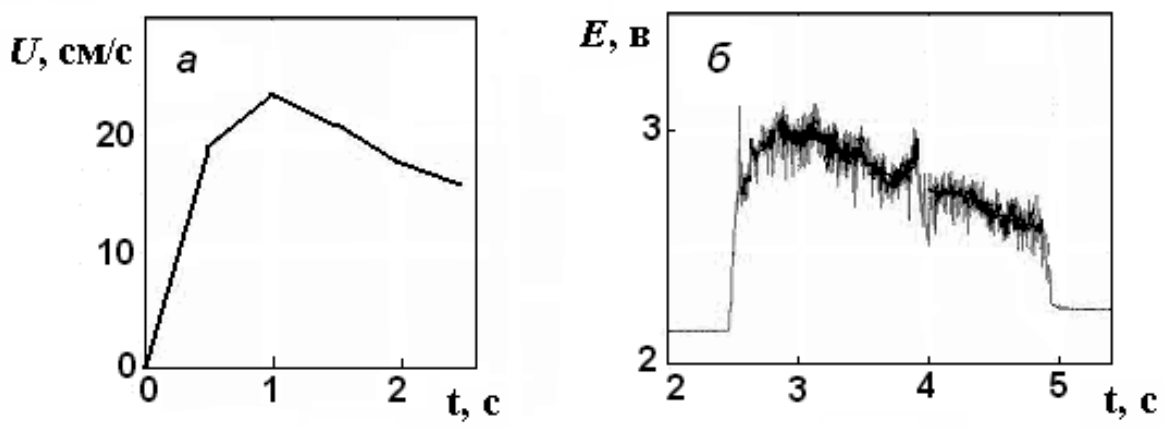

Puc. 4. Данные о скорости порошка, полученные с видеокамеры (a), и напряжение на датчике термоанемометра (б)

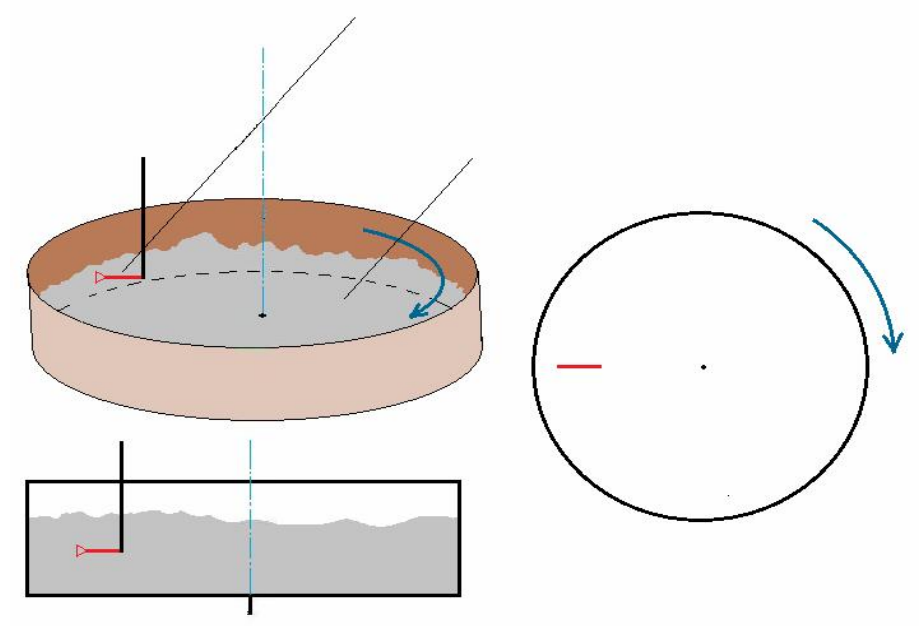

Puc. 5. Установка для тарировки датчика термоанемометра в нанопорошке
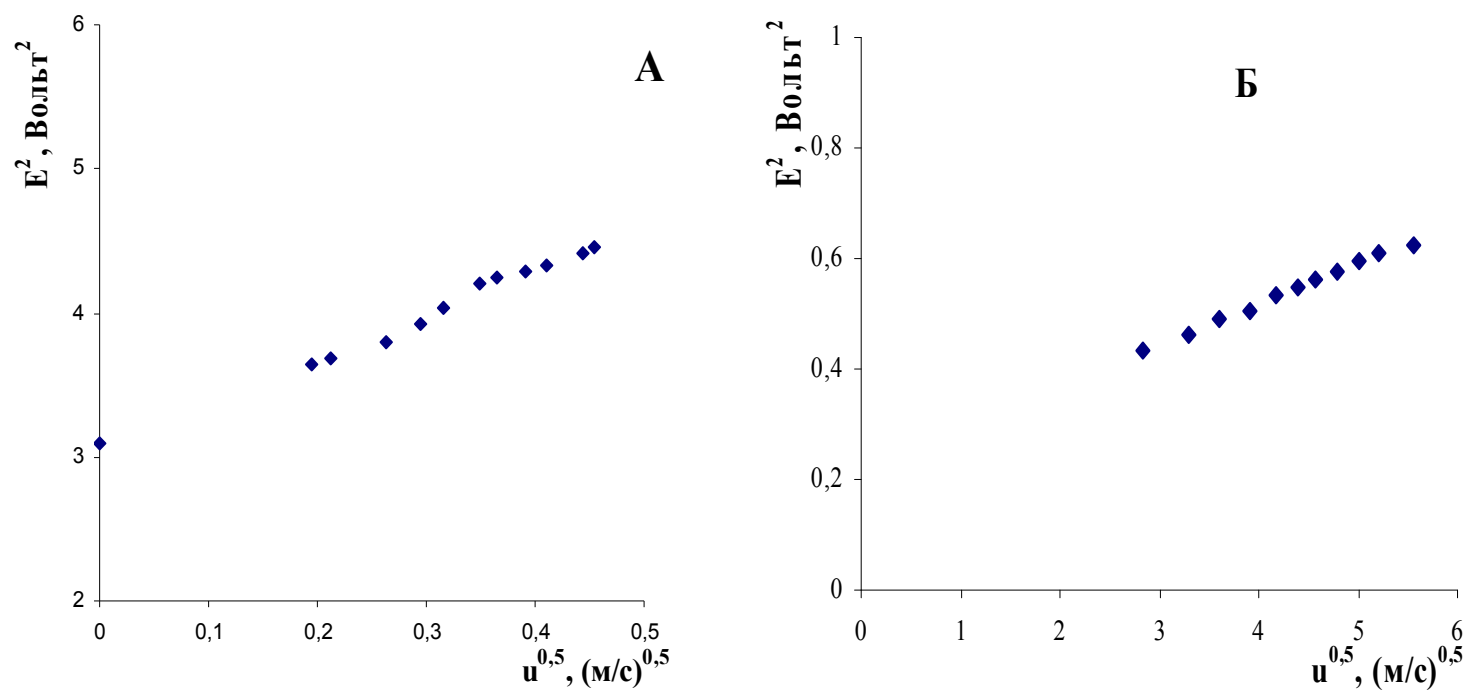

Puc. 6. Тарировочные зависимости датчика термоанемометра: $A$ - в нанопорошке; $D$ - в воздухе 
новка (рис. 5). Она включает в себя резервуap цилиндрической формы, который имеет возможность вращаться вокруг своей оси. Помещенный в такой резервуар датчик будет обтекаться потоком со скоростью, соответствующей скорости вращения резервуара.

Нанопорошок ведет себя как твердое тело, связанное с резервуаром, при низких скоростях. Стоит отметить, что нить датчика оставалась неповрежденной даже при максимальных скоростях работы установки $0,35 \mathrm{~m} / \mathrm{c}$.

Зная скорость вращения и расстояние от оси вращения до датчика, можно вычислить скорость набегающего потока. На рис. 6 приведены тарированные зависимости для одного и того же датчика термоанемометра в двух различных средах: в воздухе и в нанопорошке диоксида кремния Т-20 со средним размером частиц 20 нм и насыпной плотностью 50 г/л.

Видно, что приведенные зависимости в координатах $E^{2}$ и $u^{1 / 2}$ имеют линейный вид, что наглядно показывает схожий характер теплообмена нити с воздухом и порошком. Кроме того, из характера зависимостей видно, что теплоотвод для порошка существенно выше, чем для воздуха, примерно в 40 раз, из чего можно сделать вывод, что решающий вклад в теплообмен вносит малая по объему твердая фаза.

На рис. 7 показан характерный сигнал термоанемометра. Видно, что среднеквадратичные значения пульсаций напряжения составляют $0,12 \mathrm{~B}$, или около $6 \%$ от среднего уровня сигнала. А значит, применение термоанемометра позволяет измерить не только средние скорости, но и среднеквадратичные пульсации скорости течения порошка, а также мгновенные скорости.

Далее более подробно получены характеристики датчика термоанемометра в нанопорошке. На рис 8. видно, что зависимость квадрата напряжения от корня квадратного из скорости остается литейной при всех перегревах нити. По полученным данным была построена универсальная линейная зависимость числа Нуссельта для всех перегревов нити (рис. 9).

На рис 10 показана зависимость напряжения на выходе с термоанемометра при постоянной скорости потока от плотности порошка диоксида кремния. Найдено, что плотность не оказывает существенного влияния на по- казания датчика, а значит пульсации показаний термоанемометра обусловлены пульсациями скорости порошка.

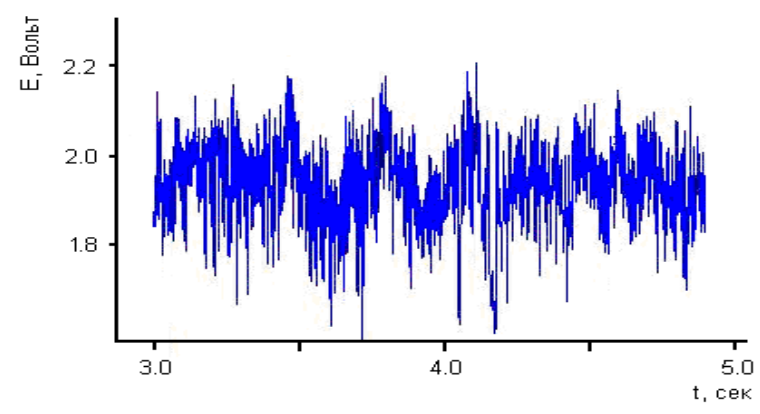

Рuc. 7. Флуктуации сигнала термоанемометра, $u=0,1 \mathrm{M} / \mathrm{c}$

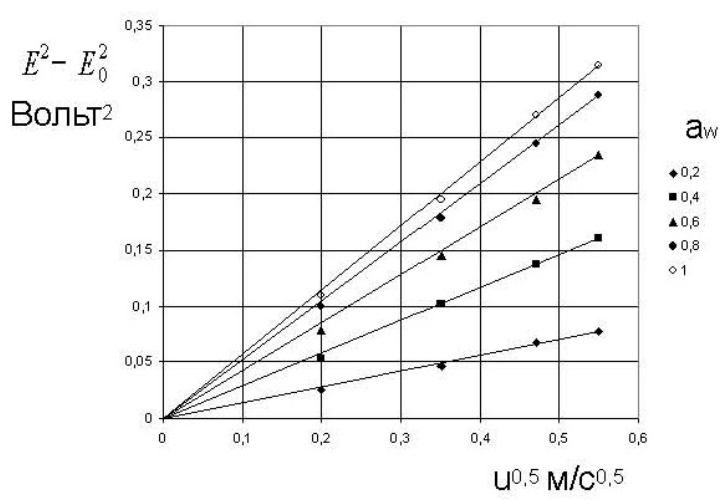

Puc. 8. Тарировочная зависимость квадрата напряжения на датчике термоанемометра от корня из скорости при различных перегревах нити

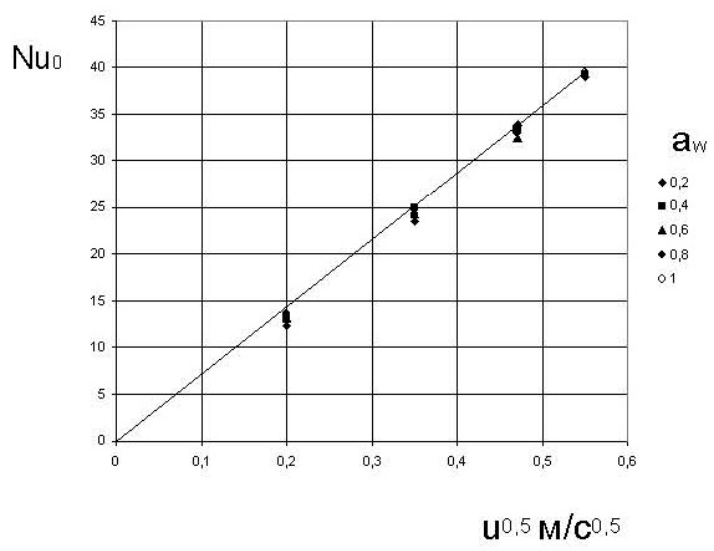

Puc. 9. Универсальная линейная зависимость числа Нуссельта для всех перегревов нити 


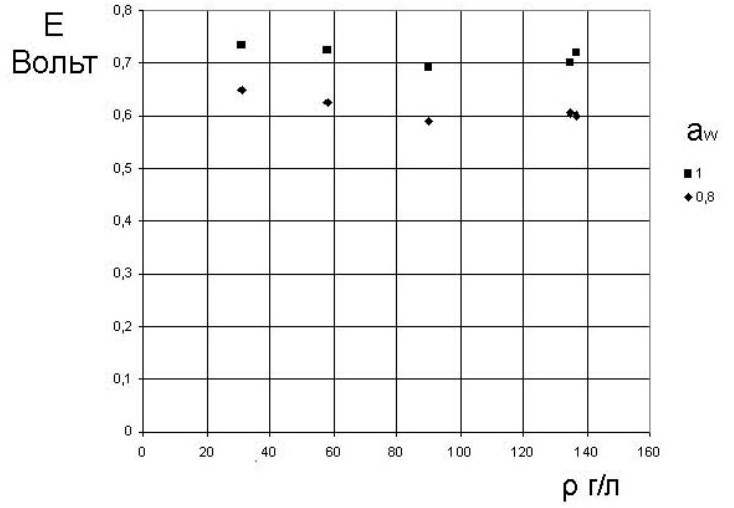

Puc. 10. зависимость напряжения на выходе с термоанемометра при постоянной скорости потока от плотности порошка

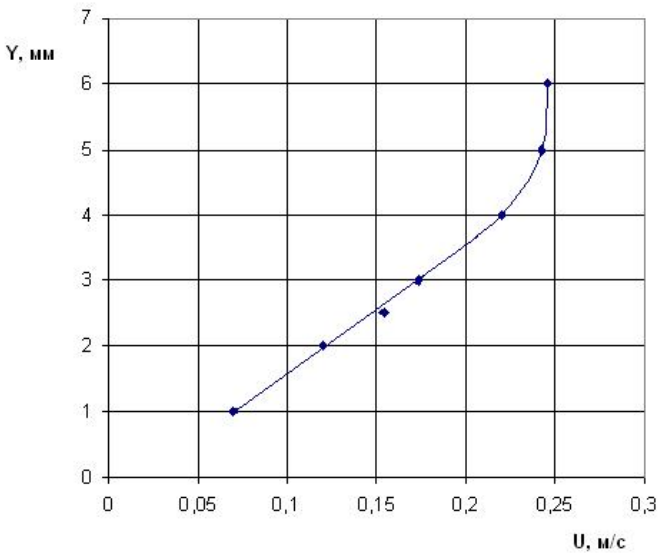

Puc. 11. Профиль средней скорости в потоке нанопорошка Таркосил при $x=260$ мм $(\operatorname{Re}=284)$

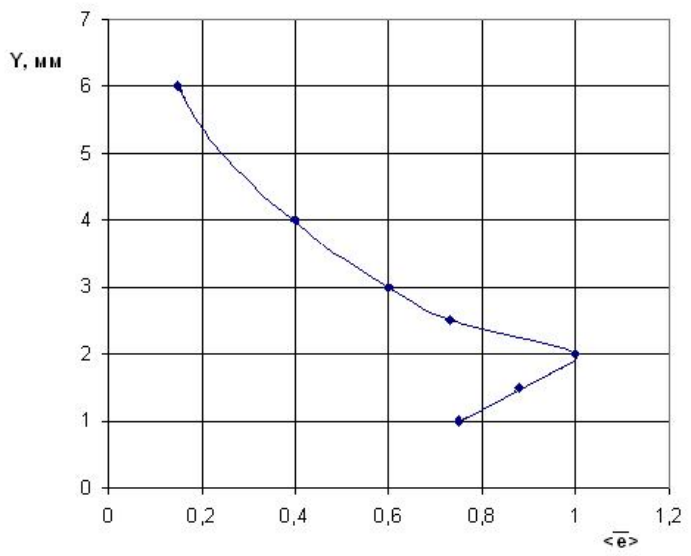

Рис. 12. Профиль обезразмеренных среднеквадратичных пульсаций напряжения в потоке нанопорошка Таркосил при $x=260$ мм $(\operatorname{Re}=284)$

\section{Исследование течения в пограничном слое на вогнутой пластине при обтекании ее нанопорошком}

Для изучения пограничного слоя в потоке нанопорошка в установку, в которой ранее проводилась тарировка, помещалась вогнутая пластина соосно оси вращения резервуара с порошком. Модель имела радиус закругления 135 мм, длину дуги 370 мм, погружалась на 7 см вглубь порошка и имела скошенную под углом $30^{\circ}$ переднюю кромку. Пластина и датчик термоанемометра остаются неподвижными относительно наблюдателя. Далее в экспериментах координата $X$ - это координата вдоль дуги модели с нулем на передней кромке, а координата $Y$ - это координата, перпендикулярная $X$, с нулем на верхней границе порошка.

Пульсационное и среднее значения напряжения на нити датчика термоанемометра записывались в ЭВМ (PC IBM) с помощью амплитудно-цифрового шестнадцатибитного преобразователя (АЦП) Е14-440 (максимальная частота 400 кГц, 16 каналов) с цифровым сигнальным процессором ADSP$2185 \mathrm{M}$ (тактовая частота работы 48 МГц). Спектры пульсаций определялись с помощью программы, любезно предоставленной А. В. Бойко. Полоса пропускания составляла 0,05 Гц. Кроме этого (для дублирования), сигнал с выхода моста подавался на вольтметр среднего напряжения РВ7-32.

В качестве рабочей среды использовался нанопорошок Таркосил Т-20, произведенный газофазным методом [2] (при помощи испарения исходного сырья пучком электронов), с удельной поверхностью $139 \mathrm{~m}^{2} /$ г и насыпной плотностью 62 г/л.

Были измерены профили средней скорости и безразмерные профили среднеквадратичных пульсаций напряжения. Видно, что эти зависимости подобны соответствующим зависимостям в течениях сплошной среды, скорость потока вблизи пластины существенно падает (рис. 11), а для среднеквадратичных пульсаций есть характерный максимум, расположенный внутри пограничного слоя, примерно на расстоянии 1,5-2 мм от поверхности пластины (рис. 12). 


\section{Акустические возмущения в нанопорошке}

В предыдущей части было показано, что с помощью датчика термоанемометра можно фиксировать флуктуации в порошке. В связи с этим интересно рассмотреть воздействия акустических возмущений на порошковую среду [15]. Для этих целей была создана установка, аналогичная установке Кундта, представляющая собой горизонтальную трубу, на половину заполненную порошком. На одном из концов трубы расположен громкоговоритель, другой конец заглушен (рис. 13).

Как и в экспериментах Кундта с обычным, не наноразмерным, порошком, на поверхности порошок-воздух в нашем случае наблюдались стоячие и бегущие волны. Также проводилось изучение распределения возмущений внутри порошка при резонансной для воздуха частоте. Для этого в установку помещался датчик термоанемометра. Датчик имел возможность перемещаться вдоль трубы. На графике представлен спектр, полученный при продольной координате, соответствующей максимальной амплитуде возмущений (рис. 14).

Термоанемометрические измерения показывают, что существуют волны внутри порошка с генерацией высших гармоник. Затем были проведены измерения групповой скорости звука в нанопорошке. Для этого использовалась полностью заполненная порошком труба, на громкоговоритель подавался прямоугольный импульс, а прохождение сигнала через порошок фиксировалось микрофоном. Групповая скорость распространения звуковой волны в наноразмерном порошке диоксида кремния составила $50-70 \mathrm{~m} / \mathrm{c}$.

\section{Выводы}

Проведены и описаны широкие исследования, касающиеся течений гранулированных сред, и акцентировано внимание на течении нанопорошков. Показано, что многие проблемы течений гранулированных сред еще не разрешены.

В статье приведен обзор работ, результаты которых свидетельствуют о необходимости постановки проблемы описания новых типов сплошных сред, содержащих большие

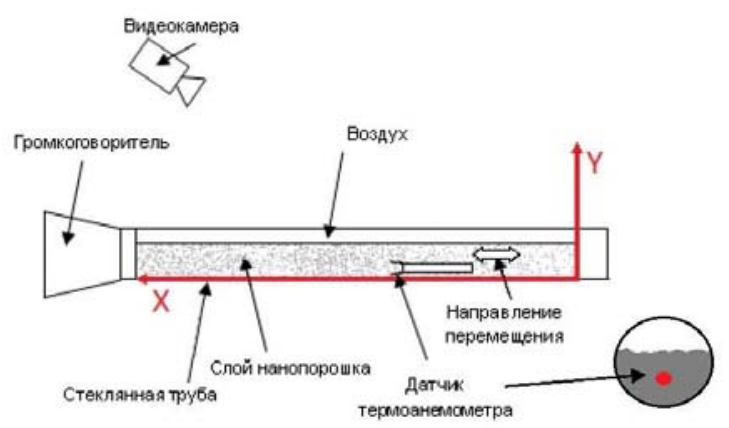

Puc. 13. Схема установки Кундта с нанопорошком

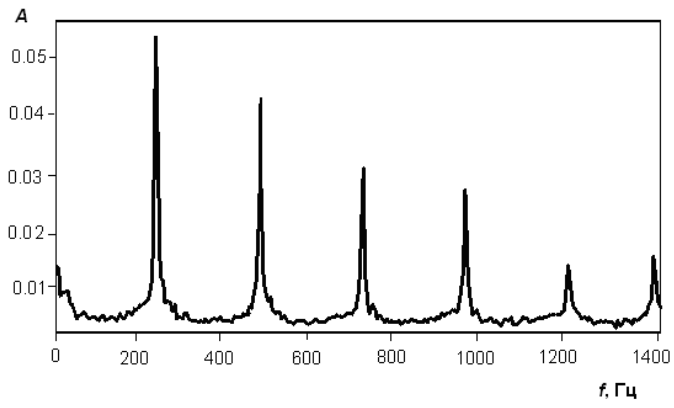

Puc. 14. Частотный спектр возмущений в максимальной точке амплитуды, измеренный термоанемометром

концентрации наноразмерных частиц. Подчеркивается важность этой проблемы для понимания современных технологических процессов, в которых образуются нанопорошки различной природы и во все возрастающей степени используются компоненты в виде наночастиц, концентрация которых достигает существенных значений. Рассмотренны примеры практически важных течений и материалов.

Предложено понятие новой сплошной среды при высокой концентрации наночастиц.

Применен термоанемометрический метод для описания течений порошковой среды, показана пригодность данного датчика для получения достоверных данных о течении гранулированной среды.

Установлено наличие пограничного слоя в нанопорошке.

\section{Список литературы}

1. AEROSIL Fymed Silica, Technical bulletin Fine Particles. 2003. № 63. 
2. Bardakhanov S. P., Korchagin A. I., Kuksanov N. K., Lavrukhin A. V., Salimov R. A., Fadeev $S$. N., Cherepkov $V$. $V$. Nanopowder Production based on Technology of Solid Raw Substances Evaporation by Electron Beam Accelerator // Mater. Sci. Eng. B. 2006. Vol. 132. P. 204-208.

3. Green R. G., Rahmat M. F., Dutton K., Evans K., Goude A., Henry M. Velocity and mass flow rate profiles of dry powders in a gravity drop conveyor using an electrodynamic tomography system // Measurement Sci. Tech. 1997. Vol. 8. No. 4. P. 429-436.

4. Uchida K., Okamoto K. Measurements of powder flow in a screw feeder by $\mathrm{x}$-ray penetration image analysis // Measurement Sci. Tech. 2006. Vol. 17. No. 2. P. 419-426.

5. Darelius A., Rasmuson A., Bjorn I. N., Folestad $S$. LDA measurements of near wall powder velocities in a high shear mixer // Chem. Eng. Sci. 2007. Vol. 62. No. 21. P. 5770-5776.

6. Mantle M. D., Sederman A. J., Gladden L. F., Huntley J. M., Martin T. W., Wildman R. D., Schattuck M. D. MRI investigations of particle motion within a three-dimensional vibro-fluidized granular bed // Powder Tech. 2008. Vol. 179. No. 3. P. 164-169.

7. Takahashi $K$. On the dynamical properties of granular mass // Geophysics Magazine. 1937. Vol. 11. P. 165-177.

8. Бардаханов С. П., Козлов С. А. Термоанемометрические измерения в течениях нанодисперсных порошков. Новосибирск, 2001.

9. Ridgway K., Rupp R. Flow of Granular Materials Down Chutes // Chem. Proc. Eng. 1970. Vol. 31. P. 82.

10. Campbell C. S., Brennen C. E. Chute Flows of Granular Materials: Some Computer Simulation // Transaction of the ASME. 1985. Vol. 52. P. 172-178.

11. Suzuki A., Takahashi H., Tanaka T. Behaviour of a particle bed in the field of vibration III. Mixing of particles in a vibrating vessel // Powder Technology. 1968. Vol. 2, iss. 2. P. 78-81.

12. Takahashi $K$. On the dynamical properties of granular mass // Geophysics Magazine. 1937. Vol. 11. P. 165-177.

13. Бардаханов С. П., Ларичкин В. В., Обанин В. В., Семенов А. Б. Исследования течений нанодисперсных сред и инженерные проблемы создания экологически чистых технологий их получения // Научный вестник НГТУ. 2005. № 3.

14. Бардаханов С. П., Лысенко В. И., Труфанов Д. Ю. Применение термоанемометра для измерений скорости течения нанопорошков // Изв. РАН. Механика жидкости и газа. 2011. № 1. С. 62-69.

15. Бардаханов С. П., Лысенко В. И., Обанин В. В., Труфанов Д. Ю. Исследование распространения акустических волн и течения в нанодисперсной среде // Теплофизика и аэромеханика. 2011. № 1. С. 27-33.

Материал поступил в редколлегию 29.03.2017

\section{S. P. Bardakhanov, D. Yu. Trufanov, A. P. Zavjalov \\ Institute of Theoretical and Applied Mechanics SB RAS \\ 4/1 Institutskaya Str., Novosibirsk, 630090, Russian Federation \\ trufanov@itam.nsc.ru \\ NEW TYPE OF MEDIUM - GASES WITH HIGH CONCENTRATION OF NANOPARTICLES}

The article gives an overview of the works, the results of which indicate the necessity of posing the problem of describing new types of continuous media containing large concentrations of nanoscale particles. The importance of this problem is underlined for understanding modern technological processes in which nanopowders of various nature are formed, and in the form of nanoparticles whose concentration reaches significant values is increasingly used. Examples of practically important flows of powder materials are considered. The concept of a new continuous medium with a high concentration of nanoparticles is proposed, and the «hot-anemometric method» is applied.

Keywords: nanopowder, gas flow, continuous medium flow, granular medium. 